\section{ACESSO À SAÚDE BUCAL PÚBLICA PELO PACIENTE ESPECIAL: A ÓTICA DO CIRURGIÃO-DENTISTA}

\author{
Access to public oral health care by special needs patients: the \\ dentist's perspective
}

\author{
Acceso del paciente especial a la salud bucal pública: la óptica \\ del cirujano-dentista
}

\section{RESUMO}

Objetivo: Conhecer, sob a ótica dos cirurgiões-dentistas, o acesso dos pacientes especiais à saúde bucal no Sistema Único de Saúde. Métodos: Estudo transversal, quantitativo, realizado em 2011 por meio da aplicação de entrevistas baseadas em questionários semiestruturados, realizadas com cirurgiões-dentistas nas Unidades Básicas de Saúde da Família (UBASF) de uma capital do nordeste brasileiro. As variáveis englobaram dados sociodemográficos, condições de trabalho e as relacionadas ao acesso e atendimento do paciente portador de necessidade especial. Os achados foram analisados com estatística adequada pelo SPSS 15. Resultados: Entrevistaram-se 44 cirurgiões-dentistas, dos quais $36(81,8 \%)$ atendiam pacientes especiais, $29(65,9 \%)$ realizavam exame clínico e orientação de higiene bucal, e 5 $(11,3 \%)$ realizavam exame clínico e encaminhavam para especialistas. Nove $(20,5 \%)$ tiveram disciplina direcionada para esse tipo de usuário. O acesso, o acolhimento e a assistência odontológica oferecidos foram aprovados por 31 cirurgiões-dentistas (70\%). Conclusão: Sob a ótica dos cirurgiões-dentista investigados, os pacientes especiais da rede pública estão tendo acesso à atenção em saúde bucal, entretanto, a resolutividade parece estar prejudicada, sendo necessário encaminhá-los a outros serviços.

Descritores: Pessoas com Deficiência; Saúde Bucal; Acesso aos Serviços de Saúde; Sistema Único de Saúde.

\section{ABSTRACT}

Objective: To examine, from the dentists' perspective, the access of special needs patients (SNP) to oral health care in the Unified Health System. Methods: Cross-sectional and quantitative study, performed in 2011 by means of interviews based on semi-structured questionnaires, carried out with dental surgeons at family primary healthcare units of a capital city in Brazil's northeast region. Study variables included sociodemographic factors, work conditions and data related to the access and utilization of dental care services by SNP. Data was analysed with proper statistics in SPSS 15. Results: Interviews were performed with 44 dentists, 36 (81.8\%) of which regularly assisted SNP; 29 (65.9\%) conducted only oral examination and oral hygiene instructions; and 5 (11.3\%) conducted oral examination and referred the patients to specialties centers. Nine (20.5\%) of them reported having attended a discipline, focused on that type of patient. The accessibility, caring and dental assistance offered to SNP were approved by 70\% of the dentists. Conclusions: From the dentists 'perspective, special needs patients in the public network have access to oral health care; resolutivity, however, seems to be undermined, being necessary to refer those to other services.

Descriptors: Disabled Persons; Oral Health; Health Services Accessibility; Unified Health System.

\section{Artigo Original}

Fabíola de Lima Gonçalves(3) Maria Vieira de Lima Saintrain ${ }^{(4)}$ Anya Pimentel Gomes Fernandes Vieira $^{(5)}$
1) University of Toronto - Toronto - Canadá

2) Prefeitura Municipal de Fortaleza Fortaleza (CE) - Brasil

3) Universidade Estadual do Ceará - UECE - Fortaleza (CE) - Brasil

4) Universidade de Fortaleza - UNIFOR Fortaleza (CE) - Brasil

5) Fundação Oswaldo Cruz - FIOCRUZ Fortaleza (CE) - Brasil

Recebido em: 11/08/2013 Revisado em: 23/10/2013 Aceito em: 23/04/2014 


\section{RESUMEN}

Objetivo: Conocer el acceso de los pacientes especiales a la salud bucal del Sistema Único de Salud desde la óptica de los cirujanosdentista. Métodos: Estudio transversal, cuantitativo realizado en 2011 a través de la aplicación de entrevistas basadas en cuestionarios semiestruturados realizadas en cirujanos-dentista de las Unidades Básicas de la Salud de la Familia (UBASF) de una capital del nordeste brasileño. Las variables incluyeron datos socio demográficos, condiciones de trabajo y los relacionados al acceso y atención del paciente portador de necesidad especial. Los hallazgos fueron analizados con estadística adecuada por el SPSS 15. Resultados: Fueron entrevistados 44 cirujanosdentista de los cuales $36(81,8 \%)$ asistían a pacientes especiales, $29(65,9 \%)$ realizaban la prueba clínica y orientación de higiene bucal y $5(11,3 \%)$ realizaban la prueba clínica y os encaminaban a los especialistas. Nueve (20,5\%) tuvieron asignatura direccionada para ese tipo de usuario. El acceso, la recepción y la asistencia odontológica ofrecidos fueron aprobados por 31 cirujanosdentista (70\%). Conclusión: Desde la óptica de los cirujanosdentista investigados, los pacientes especiales de la red pública tienen acceso a la asistencia en salud bucal, sin embargo, parece ser que la resolutividad parece estar perjudicada siendo necesario dirigirlos a otros servicios.

Descriptores: Personas con Discapacidad; Salud Bucal; Accesibilidad a los Servicios de Salud; Sistema Único de Salud.

\section{INTRODUÇÃO}

O Programa Saúde da Família (PSF), implantado em 1994 - posteriormente denominado Estratégia Saúde da Família (ESF) -, proporcionou à população brasileira conquistas nas questões relacionadas à qualidade de vida $\mathrm{e}$ cidadania. Sendo uma política do Ministério da Saúde (MS), a ESF se apresenta como uma estratégia de concretização dos princípios norteadores do Sistema Único de Saúde (SUS): universalidade, equidade e integralidade ${ }^{(1)}$.

As questões relativas ao acesso aos serviços de saúde, entretanto, são marcadas, de uma maneira geral, por situações conflituosas que angustiam trabalhadores, gestores e usuários. A realidade aponta uma dificuldade ainda maior de atendimento para o grupo de pacientes com necessidades especiais (PNE) quando a busca é por atenção em saúde bucal. A literatura assinala o baixo acesso e utilização dos serviços odontológicos, mostrando a urgente necessidade de reorganização e reestruturação desses serviços ${ }^{(2,3)}$.

Essa realidade pode ser encontrada nas Unidades Básicas de Saúde da Família (UBASF), apesar de o Ministério da Saúde expressar, através do Caderno de Atenção Básica $\mathrm{n}^{\mathrm{o}} 17$ - Saúde Bucal $^{(4)}$, que as equipes de saúde bucal na ESF deverão ser capacitadas para prestar assistência odontológica às pessoas com necessidades especiais, e estas só deverão ser encaminhadas ao atendimento especializado nos casos em que extrapolem os limites da atenção básica ${ }^{(4)}$.

$\mathrm{OMS}^{(4)}$ diz que, em Odontologia, é considerado paciente com necessidade especial todo usuário que apresente uma ou mais limitações, temporária ou permanente, de ordem mental, física, emocional, de crescimento ou médica, que o impeça de ser submetido a uma situação odontológica convencional. As razões das necessidades especiais são inúmeras e vão desde doenças hereditárias e defeitos congênitos até alterações que ocorrem ao longo da vida, como doenças sistêmicas, alterações de comportamento e o envelhecimento ${ }^{(4)}$.

Os pacientes considerados com necessidades especiais, para o atendimento odontológico, são pessoas que geralmente não têm habilidade para promover uma higiene oral satisfatória e, muitas vezes, não permitem que outras pessoas a façam de maneira adequada, por possuírem comportamento agressivo ou apresentarem movimentos involuntários, dificultando a higienização ${ }^{(5)}$.

Segundo dados recentes do Censo do Instituto Brasileiro de Geografia e Estatística (IBGE), há no Brasil cerca de 45,6 milhões de pessoas com deficiência, o que corresponde a $23,92 \%$ da população brasileira. Observase, pois, que se trata de uma grande parcela da população, sendo boa parte dela usuária do SUS, e como tal, deve ser assistida em todas as suas necessidades de saúde ${ }^{(6)}$.

Como fazer saúde da família sem prestar assistência às pessoas com deficiência ou com alguma necessidade especial? Qual seria a causa da dificuldade do acesso desses usuários à atenção básica, mais precisamente à saúde bucal? Seja por despreparo, insegurança, falta de compromisso ou preconceito, o que se sabe é que existe uma demanda "adormecida"(3-5) surgindo sem atenção adequada, tendo acesso aos serviços de Odontologia praticamente só em ocasiões de urgência, para procedimentos mutiladores. Isso denota a necessidade de se repensar a organização da atenção básica, visto que ela exerce um papel fundamental na estruturação da rede de cuidados. Entende-se que a clínica de referência é uma necessidade, pois existem casos que requerem a atenção de um especialista, e esse suporte é necessário, em consonância com a política de assegurar a assistência integral ao indivíduo em todos os seus níveis de complexidade $^{(7)}$.

Diante dessa problemática, a pesquisa teve como objetivo conhecer, sob a ótica dos cirurgiões-dentistas, o acesso dos pacientes especiais à saúde bucal no Sistema Único de Saúde.

\section{MÉTODOS}

Trata-se de um estudo transversal, de abordagem quantitativa, aplicado por meio de entrevistas com 
questionários semiestruturados, elaborados especialmente para a presente pesquisa, aos cirurgiões-dentistas lotados nas UBASF de Fortaleza-CE.

Esse município apresenta população estimada em 2.551.805 habitantes ${ }^{(7)}$, dos quais 883.132 estão cadastrados na Estratégia Saúde da Família, beneficiando 239.742 famílias ${ }^{(8)}$. Administrativamente, Fortaleza encontra-se dividida em seis Secretarias Executivas Regionais (SER), sendo alvo do presente estudo a SER VI, que atende a 49,3\% da população, com 48.358 famílias e 179.399 pessoas cadastradas ${ }^{(8)}$. A SER VI possui 20 Unidades de Atenção Básica, todas com equipes de saúde bucal (ESB), perfazendo um total de 67 cirurgiões-dentista. Além disso, conta com um Centro de Especialidades Odontológicas (CEO), que atende, entre outras especialidades, a pacientes especiais.

Todos os cirurgiões-dentistas lotados na SER VI foram procurados para participar da pesquisa, sendo excluídos do estudo os que se negaram, estavam de férias ou de licença, ou os que, após três visitas às unidades de saúde, não foram encontrados em seus locais de trabalho.

Investigaram-se as seguintes variáveis: (1) idade, (2) gênero, (3) religião, (4) naturalidade, (5) estado civil, (6) escolaridade, (7) instituição de formação, (8) tempo de formado, (9) horas trabalhadas/semana, (10) regime jurídico de trabalho, (11) tempo de atuação na ESF (12) teve disciplina/capacitação em pacientes especiais durante a graduação, (13) atende ou não o paciente portador de necessidade especial, (14) tipo de atenção dedicada ao paciente especial - esta com as seguintes subvariáveis: a) solicita que a auxiliar de saúde bucal (ASB) anote os dados e encaminhe; b) realiza o exame clínico e dá orientações de higiene bucal ao paciente e/ou cuidador; c) apenas realiza o exame clínico e encaminha; d) realiza o exame clínico e o tratamento possível; e) passa o caso para um colega; f) realiza visitas domiciliares solicitadas pelo agente comunitário de saúde (ACS), (15) na sua unidade existe assistência odontológica ao paciente especial, (16) como avalia o acolhimento na unidade de saúde, (17) acha que a unidade dá acesso ao paciente especial, (18) o que é necessário para capacitar as ESB ao atendimento do paciente especial, (19) já encaminhou algum paciente para o CEO.

Todas as entrevistas ocorreram nas UBASF, em local reservado, realizadas por uma pesquisadora treinada para fazê-las de forma uniformizada e sem influenciar os entrevistados, sendo responsável por preencher o questionário com as respostas indicadas.

Realizou-se a coleta de dados entre os meses de fevereiro e abril de 2011, sendo processados utilizando o software SPSS 15.0 (SPSS for Windows, SPSS Inc.,
Chicago, IL, USA). Fez-se análise descritiva através de tabelas de frequência para variáveis categóricas, e de média e desvio padrão para as variáveis contínuas. Analisaramse as variáveis supracitadas comparativamente por meio de testes paramétricos (teste $t$ independente, One-way ANOVA) e não paramétricos (testes Qui-Quadrado, Mann-Whitney U Test, Kruskall Wallis), de acordo com a necessidade, levando em consideração $\mathrm{p} \leq 0,05$.

Com relação aos aspectos éticos da pesquisa, o respeito ao anonimato e ao sigilo foram devidamente observados, de acordo com a Resolução 466/12 do Conselho Nacional de Saúde/Ministério da Saúde ${ }^{(9)}$. O projeto recebeu aprovação do Comitê de Ética em Pesquisa da Universidade de Fortaleza, sob o parecer n ${ }^{\circ} 344 / 2010$.

\section{RESULTADOS}

Inicialmente, a pesquisa direcionou-se para 67 cirurgiões-dentistas, atuantes em 19 das 20 Unidades Básicas de Saúde da Regional VI do município de Fortaleza. Destes, 44 responderam ao questionário $(65,7 \%)$.

Tabela I - Dados sociodemográficos dos cirurgiões-dentistas da Regional VI. Fortaleza-CE, 2011.

\begin{tabular}{lc}
\hline Variável & n ( \%) \\
\hline Sexo & $10(22,7 \%)$ \\
Masculino & $34(77,3 \%)$ \\
Feminino & \\
Estado civil & $30(68,2 \%)$ \\
Casados & $08(18,2 \%)$ \\
Solteiros & $06(13,6 \%)$ \\
Divorciados & \\
Escolaridade & $04(09,1 \%)$ \\
Graduação & $36(81,2 \%)$ \\
Especialização & $04(09,1 \%)$ \\
Mestrado & \\
Naturalidade & $34(77,3 \%)$ \\
Capital & $08(18,2 \%)$ \\
Interior & $02(04,4 \%)$ \\
Outro estado & \\
Religião & $33(75,0 \%)$ \\
Católica & $08(18,2 \%)$ \\
Evangélica & $03(06,8 \%)$ \\
Outra & \\
Universidade em que se formou & $29(65,9 \%)$ \\
Pública & $15(34,1 \%)$ \\
Privada & \\
\hline
\end{tabular}

*ESF: Estratégia Saúde da Família 
A unidade onde trabalha uma das pesquisadoras do presente artigo não participou da pesquisa, evitando viés. Do universo de 67 cirurgiões-dentista, 7 estavam de licença no momento da coleta de dados, 11 não se encontravam em seus locais de trabalho, mesmo após um número mínimo de três tentativas (visitas à unidade de saúde), e 5 se negaram a participar da entrevista, totalizando 44 cirurgiões-dentistas entrevistados.

A Tabela I apresenta os dados sociodemográficos dos entrevistados. Quanto à idade média, encontrou-se a de $36( \pm 7,3)$ anos, sendo a maioria - $34(77,3 \%)$ - do sexo feminino; $33(75,0 \%)$ pertenciam à religião católica; 34

Tabela II - Descrição da atenção oferecida aos pacientes especiais pelos cirurgiões-dentistas da Regional VI. Fortaleza-CE, 2011.

\begin{tabular}{lc}
\hline Variável & n (\%) \\
\hline Você atende o paciente com necessidade especial? Sim & $36(81,8 \%)$ \\
Solicita que a auxiliar de saúde bucal anote os dados do paciente e encaminha & - \\
Realiza o exame clínico e orienta a higiene bucal ao paciente/cuidador & $29(65,9 \%)$ \\
Apenas realiza o exame clínico e encaminha & $05(11,3 \%)$ \\
Realiza o exame clínico e o tratamento possível & $31(70,4 \%)$ \\
Passa o caso para outro colega, pois não se sente apto a atender & $03(6,8 \%)$ \\
Realiza visita domiciliar solicitada pela(o) agente comunitário de saúde & $21(47,7 \%)$ \\
\hline
\end{tabular}

Tabela III - Aspectos gerais sobre a formação dos cirurgiões-dentistas e percepção destes acerca da atenção em saúde bucal ao paciente com necessidade especial na Regional VI. Fortaleza-CE, 2011.

\begin{tabular}{lc}
\hline Variável & n(\%) \\
\hline Durante a graduação, você teve alguma disciplina que abordasse o atendimento aos pacientes especiais? & $09(20,5 \%)$ \\
Sim & $35(79,5 \%)$ \\
Não & \\
Sua unidade permite o acesso ao paciente especial? & $33(75,0 \%)$ \\
Sim & $11(25,0 \%)$ \\
Não & $31(70,4 \%)$ \\
Existe assistência odontológica a pacientes especiais em sua unidade de saúde? & $13(26,6 \%)$ \\
Sim & \\
Não & $02(4,5 \%)$ \\
Como você avalia o acolhimento da sua equipe de saúde bucal ao paciente especial? & $08(18,2 \%)$ \\
Ruim & $25(56,8 \%)$ \\
Regular & $07(15,9 \%)$ \\
Bom & $02(04,5 \%)$ \\
Ótimo & $31(67,5 \%)$ \\
Não sabe & $08(18,2 \%)$ \\
O que você acha ser necessário para melhorar o atendimento ao paciente especial por parte das equipes de saúde bucal? \\
Capacitação/aperfeiçoamento/especialização na área & $01(2,5 \%)$ \\
Outros & $04(10,0 \%)$ \\
Não sabe & $40(90,1 \%)$ \\
Não responderam & $04(09,1 \%)$ \\
Você já encaminhou algum paciente especial para os Centros de Especialidades Odontológicas? & \\
Sim & \\
Não &
\end{tabular}


$(77,3 \%)$ eram naturais de Fortaleza; $8(18,2 \%)$, do interior do Ceará e $2(4,5 \%)$, provenientes de outros estados; a maioria - $30(68,2 \%)$ - era casada; o tempo médio de formado foi de $13,1( \pm 7,2)$ anos; a média do tempo de atuação na ESF foi de $6,8( \pm 2,7)$ anos; $29(65,9 \%)$ graduaram-se em instituição de ensino superior (IES) pública; $100 \%$ dos pesquisados ingressaram no quadro de servidores municipais através de concurso público; 36 (81,8\%) fizeram especialização, destes, $13(46,4 \%)$ têm uma segunda especialização e 4 $(9,1 \%)$ têm mestrado. A especialização mais citada foi em Saúde da Família ( $\mathrm{n}=15)$, seguida pela de Saúde Pública $(n=3)$.

A descrição da atenção oferecida aos pacientes especiais pelos cirurgiões-dentista pode ser visualizada na Tabela II.

É importante observar que, apesar do grande percentual de atendimento $(81,8 \% ; n=36)$, este se limita ao exame clínico e ao tratamento possível $(70,4 \% ; n=30)$ ou ao exame clínico e orientação da higiene bucal $(65,9 \% ; n=29)$. O tratamento possível foi descrito pelos entrevistados como sendo aquele em que o CD conseguia realizar na Unidade Básica de Saúde antes de encaminhar para unidades especializadas (CEO). Os tratamentos mais descritos foram profilaxia e aplicação tópica de flúor, quase não sendo citados tratamentos restauradores, periodontais e extrações dentárias.

Quanto aos aspectos gerais sobre a formação dos cirurgiões-dentista e percepção destes acerca da atenção em saúde bucal ao paciente com necessidade especial, são descritos na Tabela III. Destaca-se que apenas 9 (20,5\%) profissionais receberam capacitação ou participaram de disciplina sobre pacientes especiais durante a graduação. Dos 44 profissionais entrevistados, 40 (90,9\%) disseram já ter encaminhado pacientes para os CEO.

Cirurgiões-dentistas com maior idade e mais tempo de formados foram aqueles que, em maior percentual, não souberam avaliar o acolhimento das suas unidades de saúde (One-way ANOVA, $\mathrm{p}=0,01 \mathrm{e} \mathrm{p}=0,008$, respectivamente). Não existe relação entre idade e atender ou não o paciente especial (Mann-Whitney $\mathrm{U}, \mathrm{p}=0,571$ ). As mulheres tendem a atender mais os PNE do que os homens (Chi-quadrado de Pearson, $\mathrm{p}=0,042$ ).

\section{DISCUSSÃO}

Devido aos avanços da ciência, houve um aumento na expectativa de vida de PNE, dessa forma, eles têm se tornado pacientes habituais de consultórios e clínicas odontológicas, procurando um acompanhamento do desenvolvimento e tratamento da dentição ${ }^{(10)}$. O atendimento odontológico a esses pacientes requer muita paciência, habilidade e carinho, pois são indivíduos carentes, excluídos de uma sociedade preconceituosa e necessitados de um atendimento especializado ${ }^{(11)}$.

Sabe-se que a atenção básica deve funcionar como porta de entrada aos demais níveis de assistência, buscando maior grau de resolubilidade das ações, viabilizando cuidados de promoção da saúde, prevenção, tratamento e reabilitação de doenças e agravos. A Unidade Básica de Saúde deve se constituir como o local por excelência do atendimento à pessoa com deficiência, dada sua proximidade geográfica e sociocultural com a comunidade circundante ${ }^{(12,13)}$. Portanto, é da responsabilidade da ESF a garantia do acesso universal aos serviços de saúde.

Para tanto, a formação dos profissionais da ESF, seja durante sua graduação ou pós-graduação, é essencial a uma atenção adequada aos PNE, pois é necessário que os profissionais estejam sensibilizados a assistir ao seu paciente em sua integralidade ${ }^{(14)}$. A problemática da pessoa com deficiência e/ou incapacidades, deslocada dos pressupostos organicistas, ao considerar apenas o corpo patológico, desvalendo o corpo relacional (inserido no espaço familiar e social), não favorece um cuidado humanizado e apropriado para esse grupo populacional ${ }^{(14)}$. A concepção de um olhar diferenciado perpassa a clínica propriamente dita, ocorrendo de forma mais eficiente quando os profissionais são conhecedores da realidade da população assistida e têm consciência do papel que exercem na integralidade do cuidado, além de sua importância profissional para o desenvolvimento da saúde pública ${ }^{(14-16)}$.

A escolha pela especialização em Saúde da Família ou Saúde Pública por uma parte considerável dos entrevistados da presente pesquisa é vista, pelos autores, como fato positivo. De acordo com o Ministério da Saúde, mudanças relacionadas à melhora na qualidade das consultas e resolutividade, na abordagem e compreensão da família atendida, e na organização do trabalho são observadas em profissionais que participam de formação (como especialização e processos de educação permanente) $)^{(17)}$.

Existe ainda uma deficiência nos currículos dos cursos de Odontologia em relação à formação profissional para o atendimento das pessoas com deficiências físicas e mentais ${ }^{(18)}$, fato evidenciado no presente estudo e que fortalece os resultados obtidos, pois os cirurgiões-dentista afirmaram que não se sentiam seguros para o atendimento, indicando o paciente para outros profissionais e enfatizando a necessidade de concretizar formação nessa área, haja vista que somente $20,5 \%$ dos dentistas pesquisados disseram ter tido disciplina ou capacitação na área de pacientes especiais durante seus cursos de graduação.

O Ministério da Saúde tem percebido que, além de investir na especialização dos profissionais já graduados, são necessárias mudanças na formação da 
graduação universitária, como investimentos públicos no financiamento de projetos e programas que alterem os currículos dos cursos de graduação das IES, para que se aproximem mais da realidade do trabalho no SUS ${ }^{(17)}$, como o Programa de Educação pelo Trabalho para a Saúde (PETSaúde) e o Programa Nacional de Reorientação da Formação Profissional em Saúde (Pró-Saúde), desenvolvidos pelo Departamento de Gestão da Educação na Saúde (DEGES) (19). Um dos motivos para o não atendimento dos PNE se dá pela falta de treinamento/capacitação dos profissionais para tal $^{(14)}$, fato observado também na presente pesquisa.

Pacientes portadores de deficiência física e mental podem apresentar, para os cirurgiões-dentistas, algumas dificuldades no seu manejo e no tratamento odontológico ${ }^{(20-24)}$. A saúde bucal do PNE é, geralmente, comprometida. A esse comprometimento inicial (causado por questões intrínsecas de alguns desses pacientes), somase o fato de poucos cirurgiões-dentistas se interessarem em atendê-los ${ }^{(11)}$, comprometendo o acolhimento das demandas odontológicas dessa população e gerando um quadro complexo e desafiador a ser vencido pelo sistema público de saúde ${ }^{(18)}$. Os profissionais e o serviço de saúde atuam em um determinado contexto, com seus valores, finalidades e práticas $^{(25)}$.

Observou-se que a maioria dos cirurgiões-dentista informou realizar exame clínico, higiene bucal ou tratamento possível nessa população. Apesar de ser um dado aparentemente positivo, é necessário discutir o que significa "tratamento possível", podendo ser uma profilaxia ou aplicação de flúor, não realizando procedimentos mais complexos, como restaurações, raspagens e/ou exodontias, conforme a necessidade do paciente. Dessa forma, temse uma população que precisa de atenção e continua com necessidades odontológicas não atendidas, apesar de ter sido "assistida" por um profissional.

A reflexão sobre os achados da atual pesquisa, que apresentam aprovação dos profissionais entrevistados de $75 \%, 57 \%$ e $70 \%$ aos questionamentos acerca do acesso, acolhimento e assistência odontológica, respectivamente, demonstra um contrassenso com a necessidade acumulada e a pouca resolutividade da atenção prestada. Entretanto, há uma preocupação por parte dos profissionais com a assistência prestada ao PNE, uma vez que demonstram interesse em ter capacitações $(n=31 ; 70,5 \%)$ direcionadas a essa clientela específica. É importante frisar que a capacitação deve incluir, além de formação clínica, aspectos relacionados ao acolhimento e a integralidade do cuidado.

O acolhimento é uma ferramenta da Política Nacional de Humanização (HUMANIZASUS), lançada em 2004 pelo Ministério da Saúde ${ }^{(26)}$. Acesso, integralidade, humanização e equidade são temas constantes na bibliografia científica que trata da organização e qualidade dos serviços de saúde no SUS. A especialização extremada das intervenções e a hierarquização dos serviços são consideradas por vários autores como fatores que contribuem para limitar as possibilidades de universalidade ${ }^{(16)}$, que, juntamente com integralidade e equidade, compõem os princípios doutrinários dos $\mathrm{SUS}^{(27,28)}$.

O princípio da equidade do SUS pressupõe que se ofereça mais assistência a quem mais dela necessitar ${ }^{(29)}$. Logo, o paciente especial, por suas peculiaridades, é um usuário que deveria ser mais bem acolhido pelas unidades de saúde, com um esforço por parte das equipes de saúde da família e equipes de saúde bucal, para a resolução de suas demandas ${ }^{(30)}$. Infelizmente, isso não foi observado, pois ainda há limitação do tratamento realizado nesses pacientes - só é feito o tratamento possível - e existe um percentual importante de encaminhamentos aos CEO.

Contextualizando o contrassenso supramencionado, uma pesquisa sobre as dificuldades encontradas pelos responsáveis/cuidadores para manter a saúde bucal em PNE comprovou que as maiores dificuldades apontadas pelos pais para manter a saúde bucal dos seus filhos foram: encontrar um dentista que atenda seu filho e o alto custo do tratamento odontológico ${ }^{(24)}$. O atendimento odontológico dos pacientes especiais na rede pública é prejudicado por fatores como situação socioeconômica baixa, necessidade de grandes deslocamentos, dificuldades de transportes, além do tempo despendido nos diversos atendimentos de reabilitação paralelos ao tratamento odontológico, juntamente com a predisposição que esses pacientes têm de adoecer, associados à falta de compreensão, interesse e/ou resistência dos pais sobre a importância da saúde bucal $^{(16,18-21)}$.

Como mencionado anteriormente, observou-se no presente estudo uma limitação no tipo de atendimento dispensado a essa clientela pelos profissionais entrevistados. Destes, 90,9\% disseram já ter encaminhado PNE para os CEO. A portaria $n^{\circ}$ 599/GM, de 23 de março de 2006, que define a implantação dos Centros de Especialidades Odontológicas estabelece que todo CEO deve realizar atendimento a $\mathrm{PNE}^{(26)}$. Procedimentos da atenção básica, como exodontias simples e dentística básica, só se justificam nos atendimentos do CEO se forem realizados em pacientes especiais. O Caderno de Atenção Básica n ${ }^{\circ} 17$ - Saúde Bucal afirma que a Unidade Básica de Saúde deverá acolher avaliar e tratar, sempre que possível, paciente com limitações motoras, deficiência visual, auditiva ou de fala, gestantes, bebês, diabéticos, cardiopatas, idosos, HIV positivos, pacientes com disfunção renal, defeitos congênitos e transplantados, sem outras limitações. O encaminhamento deveria ser feito após avaliação e/ou tentativas de atendimento na atenção básica, acompanhado 
de relatório detalhado justificando a referência, assinado pelo profissional ${ }^{(4)}$.

É importante mencionar que a especialização em Pacientes com Necessidades Especiais é considerada relativamente nova, tendo sido regulamentada somente em 2001 pelo Conselho Federal de Odontologia e oferecida pela Associação Brasileira de Odontologia em Fortaleza, somente a partir de $2005^{(10)}$, cujo fato pode limitar a oferta de profissionais capacitados no mercado de trabalho público e privado.

A avaliação como instrumento de gestão em saúde tem como propósito fundamental dar suporte aos processos decisórios no âmbito do SUS e deve auxiliar na identificação de problemas e reorientação de ações e serviços desenvolvidos, bem como avaliar a incorporação de novas práticas de saúde nos processos de trabalho e mensurar o impacto das ações implementadas pelos serviços e programas sobre o estado de saúde da população usuária do sistema ${ }^{(31)}$. Há estudo utilizando a visão do dentista sobre a atenção a saúde ${ }^{(32,33)}$, demonstrando que a perspectiva desse profissional é importante para o melhor entendimento da realidade do serviço. O presente estudo pretende auxiliar na questão do acesso aos serviços públicos pelos PNE.

Entretanto, é necessário mencionar que houve limitação neste trabalho, haja vista que apenas a visão do cirurgiãodentista foi investigada. Seria interessante a obtenção de informações dos próprios pacientes e de seus cuidadores, além dos gestores do serviço, para uma visão mais ampla e completa da realidade do acesso. Outra limitação é que se investigou apenas uma Regional do município de Fortaleza, o que pode não ser a realidade do município e do estado do Ceará como um todo.

\section{CONCLUSÃO}

Sob a ótica dos cirurgiões-dentistas investigados, os PNE da rede pública estão tendo acesso à atenção em saúde bucal, entretanto, a resolutividade desta parece estar prejudicada devido ao fato de os cirurgiões-dentistas não atenderem completamente as necessidades desse grupo de pacientes, tendo que encaminhá-los a outros serviços.

\section{REFERÊNCIAS}

1. Medeiros Júnior A, Roncalli AG. Políticas de saúde no Brasil. In: Ferreira MAF, Roncalli AG, Lima KC. Saúde bucal coletiva: conhecer para atuar. Natal: UFRN; 2004. p. 249-59.

2. Barros AJD, Bertoldi AD. Desigualdades na utilização e no acesso a serviços odontológicos: uma avaliação em nível nacional. Ciênc Saúde Coletiva. 2002;7(4):70917.
3. Pinheiro RS, Torres TZG. Uso de serviços odontológicos entre os Estados do Brasil. Ciênc Saúde Coletiva. 2006;11(4):999-1010.

4. Ministério da Saúde (BR). Cadernos de Atenção Básica. Brasília: Ministério da Saúde; 2006. (Caderno de Atenção Básica, 17).

5. Resende VLS, Castilho LS, Souza ECV, Jorge WV. Atendimento odontológico a pacientes com necessidades especiais. In: Anais do $8^{\circ}$. Encontro de Extensão da UFMG. Belo Horizonte; 2010 [acesso em 2010 Ago 28]. Disponível em: http://www.ufmg.br

6. Instituto Brasileiro de Geografia e Estatística IBGE. Censos Demográficos [acesso em 2014 Maio 2]. Disponível em: http://www.ibge.gov.br/home/ estatistica/populacao/censo2010/calendario.shtm

7. Fundo Nacional de Saúde (BR) [internet]. Brasília: Ministério da Saúde; 2014 [acesso em 2014 Abr 29]. Disponível em: http://www.fns.saude.gov.br

8. Secretaria Municipal da Saúde de Fortaleza. Relatório de Gestão 2010: saúde, qualidade de vida e a ética do cuidado. Fortaleza; 2011 [acesso em 2014 Maio 2]. Disponível em: http://www.sms.fortaleza.ce.gov.br

9. Ministério da Saúde (BR), Conselho Nacional de Saúde. Resolução no 466, de 12 de dezembro de 2012. DOU. 2013 Jun 13; no 12 Seção 1; p. 59 [acesso em 2013 Nov 4]. Disponível em: http://conselho.saude. gov.br/resolucoes/2012/Reso466.pdf

10. Arrais LA. Disponibilidade ao atendimento de pacientes especiais pelos odontopediatras de Fortaleza-CE [monografia de especialização]. Fortaleza: Associação Brasileira de Odontologia; 2007.

11. Rocha LL. Acessibilidade das pessoas com deficiência aos serviços públicos odontológicos em Fortaleza-Ce [dissertação]. Fortaleza: Universidade de Fortaleza; 2012 [acesso em 2014 Maio 2]. Disponível em: uol01. unifor.br/oul/conteudosite/F1066348885/Dissertacao. pdf

12. Cunha ABO, Vieira-da-Silva LM. Acessibilidade aos serviços de saúde em um município do Estado da Bahia, Brasil, em gestão plena do sistema. Cad Saúde Pública. 2010;26(4):725-37.

13. Rocha EF, Kretzer MR. Ações de reabilitação de pessoas com deficiência na estratégia da saúde da família da Fundação Zerbini e Secretaria Municipal de Saúde de São Paulo - Região Sudeste - Sapopemba/ Vila Prudente - período 2000/2006. Rev Ter Ocup. 2009;20(1):59-67. 
14. Bonato LL, Lopes AMS, Silva CM, Itner RG, Silva ACH. Situação atual da formação para assistência de pessoas com necessidades especiais nas faculdades de odontologia no Brasil. ClipeOdonto. 2013;5(1):10-5.

15. Silva TAM, Fracolli LA, Chiesa AM. Professional trajectory in the family health strategy: focus on the contribution of specialization programs. Rev Latinoam Enferm. 2011;19(1):148-55.

16. Resende VLS, Castilho LS, Viegas CMS, Soares MA. Atendimento odontológico a pacientes com necessidades especiais. In: Anais do $7^{\circ}$ Encontro de Extensão da Universidade Federal de Minas Gerais. Belo Horizonte; 2004 [acesso em 2011 Maio 21]. Disponível em http://www.ufmg.br/congrext/Saude/ Saude32.pdf

17. Ministério da Saúde (BR), Secretaria de Gestão do Trabalho e da Educação na Saúde. Gestão da Educação [Internet]. Brasília: Ministério da Saúde; 2014 [acesso em 2014 Maio 2]. Disponível em: http://portalsaude. saude.gov.br/index.php?option=com_content $\& v i e w=a$ rticle $\&$ id $=9276 \&$ Itemid $=473$

18. Bhowate R, Dubey A. Dentofacial changes and oral health status in mentally challenged children. J Indian Soc Pedod Prev Dent. 2005;23(2):71-3.

19. Camargo MA, Antunes JL. Untreated dental caries in children with cerebral palsy in the Brazilian context. Int J Paediatr Dent. 2008;18(2):131-8.

20. Guerreiro PO, Garcias GL. Diagnóstico das condições de saúde bucal em portadores de paralisia cerebral do município de Pelotas, Rio Grande do Sul, Brasil. Ciênc Saúde Coletiva. 2009;14(5):1939-46.

21. Huang ST, Hurng SJ, Liu HY, Chen CC, Hu WC, Tai $\mathrm{YC}$, et al. The oral health status and treatment needs of institutionalized children with cerebral palsy in Taiwan. J Dent Sci. 2010; 5(2):75-89.

22. Othero MB, Dalmaso ASW. Pessoas com deficiência na atenção primária: discurso e prática de profissionais em um centro de saúde-escola. Interface Comun Saúde Educ. 2009;13(28):177-88.

23. Ministério da Saúde (BR). Humanizasus: acolhimento com avaliação e classificação de risco: um paradigma ético estético no fazer saúde. Brasília: Ministério da Saúde; 2009 [acesso em 2011 Jun 17]. Disponível em: bvsms.saude.gov.br/bvs/publicacoes/acolhimento.pdf

24. Marra PS. Dificuldades encontradas pelos responsáveis para manter a saúde bucal de portadores de necessidades especiais [dissertação]. Duque de Caxias: Universidade do Grande Rio; 2007 [acesso em 2011 Maio 20].
Disponível em: http://www.dominiopublico.gov.br/ pesquisa/DetalheObraForm.do?select_action $=\& \mathrm{co}_{-}$ obra $=82660$

25. Sampaio EF, César FN, Martins MGA. Perfil odontológico dos pacientes portadores de necessidades especiais atendidos no instituto de previdência do Estado do Ceará. 2004. Rev Bras Promoç Saúde 2004;17(3):127-34.

26. Ministério da Saúde (BR). Portaria n 599 de 23 de Março de 2006. Define a implantação de Especialidades Odontológicas (CEOs) e de Laboratórios Regionais de Próteses Dentárias (LRPDs) e estabelecer critérios, normas e requisitos para seu credenciamento [acesso em 2014 Maio 2]. Disponível em: http://www.brasilsus. com.br

27. Brasil. Lei 8.080/90 Brasília: Diário Oficial da União, 1990.

28. Brasil. Lei 8.142/90 Brasília: Diário Oficial da União, 1990.

29. Teixeira CF, Solla J, organizadores. Modelo de atenção à saúde: promoção, vigilância saúde da família. Salvador: CEPS-ISC - EDUFBA; 2006.

30. Paim JS. Reforma Sanitária Brasileira: contribuição para compreensão e crítica. Salvador: EDUFBA; 2008

31. Vieira-da-Silva LM. Conceitos, abordagens e estratégias para a avaliação em saúde. In: Hartz ZMA, Vieira-daSilva LM, organizadores. Avaliação em saúde: dos modelos teóricos à prática na avaliação de programas e sistemas de saúde. Salvador: EDUFBA; Rio de Janeiro: FIOCRUZ; 2005. p. 15-39.

32. Fadel MAV, Regis Filho GI. Percepção da qualidade em serviços públicos de saúde: um estudo de caso. Rev Adm Pública. 2009;43(1):7-22.

33. Cunha AR, Bavaresco CS, Carrard VC, Lombardo EM. Atrasos nos encaminhamentos de pacientes com suspeita de câncer bucal: percepção dos cirurgiões dentistas na atenção primária à saúde. J Bras Telessaúde. 2013;2(2):66-74.

\section{Endereço de correspondência:}

Herenia Lawrence

University of Toronto

563 Spadina Crescent

Toronto - Ontario M5S 2J7 - Canadá

E-mail: herenia.lawrence@dentistry.utoronto.ca 